\title{
Weekly $v s$. tri-weekly cisplatin based chemoradiation in carcinoma cervix: a prospective randomized study of toxicity and compliance
}

\author{
Aradhana Katke, R Nanda, Thejaswini B, Tanveer Pasha, Giri G V, Govind Babu, Yashwant Pawar \\ Department of Radiation Oncology, Kidwai Memorial Institute of Oncology, Bangalore, India
}

\begin{abstract}
Background: Addition of chemotherapy to radiation has improved 5 -year survival by $6 \%$. However, the optimal dose and schedule of concurrent cisplatin is not well defined, though widely accepted practice is the weekly schedule of $40 \mathrm{mg} / \mathrm{m}^{2}$ for 5 weeks. Repeated admissions for weekly cisplatin drain the limited resources in high volume centres. We intended to study the compliance and toxicity of two cisplatin schedules in our patients diagnosed with carcinoma cervix.

Materials and methods: Between 2007-2011, 212 patients, histologically proven squamous cell carcinoma with stages IIB to IIIB were randomized into two arms. All patients were planned for external beam radiotherapy 45 Gy $/ 25$ frs over 5 weeks followed by Intracavitary or Interstitial brachytherapy to a total BED dose of 75-85 Gy. Single agent cisplatin given concomitantly, was scheduled weekly ( $40 \mathrm{mg} / \mathrm{m}^{2} / \mathrm{cycle}, 5$ cycles) in an arm A and three weekly ( $100 \mathrm{mg} / \mathrm{m}^{2} / \mathrm{cycle}, 2$ cycles) in an arm B. Toxicity and compliance were evaluated weekly according to the RTOG guidelines. Analysis of the compiled data was done using SSPS version 20.

Results: Of the evaluable 212, 109 patients received weekly cisplatin chemotherapy and 103 patients received three weekly cisplatin. The most common acute toxicity observed was grade I-II leucopoenia. The upper and lower gastrointestinal reactions were high in three weekly arms, which was statistically significant $(57 \%$ and $42.7 \%, p<0.05)$. Proctitis was observed in $10 \%$ of patients in both of the arms and only two patients had Gr1 Cystitis after 6 months of treatment.

Conclusions: Tri-weekly cisplatin based concurrent chemoradiation can be adopted in high volume centres with manageable haematological and gastrointestinal acute toxicities.
\end{abstract}

Key words: cancer cervix; concurrent chemoradiation; toxicities; weekly cisplatin; tri-weekly cisplatin

Rep Pract Oncol Radiother 2021;26(6):948-954

\section{Introduction}

Cervical cancers are the most common gynaecological malignancies in India. The worldwide cervical cancer estimates by the GLOBOCAN statistics revealed 569,847 (3.2\% of all sites) new cases in 2018 with approximately 311,365 (3.3\% of all sites) deaths attributed to $7.5 \%$ of all female cancer deaths
[1]. According to the National Health Portal India, 122,844 of 436.76 million women are annually diagnosed with cervical cancer that remains the second most common cancer in India [2].

Radical concurrent chemoradiation is the current standard of treatment in locally advanced cervical cancer. The five-year overall survival has improved by $6 \%$ with the addition of chemothera-

Address for correspondence: Dr. R Nanda, Associate Professor, Department of Radiation Oncology, Kidwai Cancer Institute, Hosur Road, Bangalore 560029, tel: +91-9341241084; e-mail: nanda.ramanand@gmail.com 
py to radiotherapy according to the meta-analysis group from the Medical Research Council (MRC), United Kingdom (UK) [3]. Historically, the 5-year overall survival (OS) and progression-free survival (PFS) ranged from $62 \%$ to $73 \%$ and $57 \%$ to $67 \%$, respectively [4-7]. Acute toxicities were reported to be $45 \%$ (grade 3), $12 \%$ (grade 4 ) with multi-drug combination therapy, and 35\% (grade 3 and more) with weekly cisplatin $[4,5,8]$. Data regarding late toxicities are scarce and the reports ranged from $13-16 \%$ in both single and multi-drug regimes [5-9]. Cisplatin alone is proven to be superior to non-cisplatin multi-drug chemotherapy and is equally effective with lesser toxicity in combination with radiotherapy [4].

The optimal dose and scheduling of concurrent cisplatin have not been well defined. The widely accepted regimen is $40 \mathrm{mg} / \mathrm{m}^{2}$ of cisplatin, administered weekly for 5-6 cycles, concomitantly with radiation therapy [10]. Three-weekly administration of cisplatin is routinely used in the treatment of head and neck cancers, which are histologically and biologically similar to cervical cancer [11-14]. Few studies have compared concurrent weekly administration of cisplatin versus a cytotoxic dose administered once in 3 weeks [15-18]. Repeated admissions for weekly cisplatin drain the resources in the centre like ours having a large patient load. Therefore, we intended to compare the toxicity and compliance of three-weekly schedules of cisplatin to that of a weekly schedule in our patients diagnosed with locally advanced cervical cancer.

\section{Materials and methods}

Between 2007-2011, 212 patients with histopathologically proven squamous cell carcinoma of the cervix having normal haematological, renal (glomerular filtration rate), hepatic, cardiac (electrocardiography and two-dimensional [2D] echo), audiometry parameters were randomized to receive weekly or three-weekly cisplatin along with radical radiotherapy. The radiological evaluation such as chest radiograph and computed tomography scan of abdomen and pelvis was performed to assess the extent of disease. Cystoscopy and colonoscopy were performed to rule out infiltration into the bladder and rectum in case of suspicious infiltration. $\mathrm{Pa}$ tients detected with para-aortic nodes of short-axis diameter of $>1 \mathrm{~cm}$, pelvic nodes greater than $3 \mathrm{~cm}$, and frank infiltration of the bladder or rectum on CT scan were excluded from the study. Patients with uncontrolled diabetes mellitus/hypertension, active tuberculosis, human immunodeficiency virus/hepatitis $\mathrm{B}$ surface antigen were excluded. $\mathrm{Pa}-$ tients having haemoglobin of less than $10 \mathrm{gm} / \mathrm{dL}$ at diagnosis were transfused with packed red blood cells to increase the haemoglobin to a minimum of $10 \mathrm{gm} / \mathrm{dL}$ and the same was maintained during the course of treatment.

\section{Radiotherapy}

All the patients were treated with radical intent with external beam radiation to a total dose of 45 Gy in 25 fractions over 5 weeks [equivalent total doses in 2-Gy fractions (EQD2) of $44.25 \mathrm{~Gy}$ and biological effective dose (BED) of 53.1Gy to the tumor] on telecobalt with either anteroposterior (AP) parallel opposed fields or four-field box technique depending on AP-posteroanterior (PA) separation of $20 \mathrm{~cm}$ or more. The target volume included gross tumour, uterus, parametria, vagina, and regional lymph nodes. In the two-field AP-PA technique, treatment was delivered by the source to skin distance of $80 \mathrm{~cm}$ with superior border placed at the L4-5 junction, inferior border $2 \mathrm{~cm}$ beyond the lowest extension of the lesion or base of the obturator foramen, and $1.5 \mathrm{~cm}$ beyond the pelvic brim on either side.

In the four-field box technique, limited cut CT scans were obtained, and the target volume was traced in the ratio of $1: 1$. The treatment was delivered by the source-axis distance technique. The bladder protocol was followed in all the patients with advice to empty the bladder and drink half a litre of water half an hour before the treatment.

Intracavitary brachytherapy was delivered using modified Fletcher-Suit manual after-loading low dose rate (Cs137) applicators to a total dose of 30 Gy to point A. ICRU 38 guidelines were followed in the planning and treatment of brachytherapy. Patients unsuitable for intracavitary brachytherapy were treated with an interstitial implant using Syed-Neblett Gynae-3 template with a dose of 21 Gy in three fractions (EQD2 29.76 Gy and BED of 35.7 Gy to the tumor), with remote after-loading high dose rate (Ir192). Interstitial brachytherapy was planned with a $2 \mathrm{D}$ abacus or three-dimensional brachyvision (Eclipse v8.0) planning system. The 
total bladder and rectal doses were restricted to a maximum of $70 \mathrm{~Gy}$ and $65 \mathrm{~Gy}$, respectively.

Weekly toxicity was assessed according to the Radiation Therapy Oncology Group and managed accordingly. Overall treatment time was intended to be 49-51 days from the start of pelvic irradiation.

\section{Chemotherapy}

One hundred and nine patients were randomized to a weekly cisplatin arm ( $\operatorname{arm~A)}$ and 103 to a tri-weekly arm (arm B). Patients in arm A received $40 \mathrm{mg} / \mathrm{m}^{2}$ of concurrent cisplatin once a week and arm $B$ received $100 \mathrm{mg} / \mathrm{m}^{2}$ of concurrent cisplatin once in 3 weeks. Cisplatin was delivered as an intravenous infusion over $2 \mathrm{~h}$ before radiation after adequate pre-medication which included dexamethasone $16 \mathrm{mg}$, pantoprazole $40 \mathrm{mg}$, ondansetron 8 $\mathrm{mg}$, mannitol $10 \mathrm{gm}$, potassium chloride $20 \mathrm{mEq}$, and magnesium sulphate $20 \mathrm{mg} / \mathrm{mL}$. All patients were hydrated with $1 \mathrm{~L}$ of normal saline before and after cisplatin infusion. When the total calculated cisplatin dose exceeded $70 \mathrm{mg} /$ day, the total dose was divided and delivered in 2 consecutive days, in both arms. To assess the tolerability and toxicities to chemotherapy, we decided to administer a minimum of four cycles and a maximum of five cycles of cisplatin in the weekly arm and two cycles in the three-weekly arm. The haematological and biochemical acute toxicities were evaluated twice weekly using the CTCAE version 3.0.

This study was approved by the scientific review board and ethical committee of our institute.

\section{Statistical analysis}

Descriptive statistics were used to express data. Association between categorical clinical variables and the type of chemotherapy was studied using Fisher's exact test owing to small frequencies. Clinically apparent disease at 6 weeks after the completion of treatment was classified as having 0 months of the disease-free interval. Statistical analysis was performed using the SPSS inc. Version 16 and $\mathrm{R}$ software.

\section{Results}

The median follow-up was 46.6 months (range between 24-122 months) at the time of analysis. The patient characteristics are listed in Table 1. The median overall treatment time was 46 and 47 days in $\operatorname{arm~A}$ and $\operatorname{arm~B,~respectively.~All~the~patients~}$ in our study received radiotherapy as planned. Chemotherapy given currently, $66.07 \%$ in weekly and $78.64 \%$ in tri-weekly completed the planned dose of cisplatin. The mean cumulative dose of cisplatin was higher in three-weekly arm $262.62 \mathrm{mg} v s$. $227.16 \mathrm{mg}$ weekly.

The acute toxicities observed are as enlisted in Table 2. Leucopenia was the most common and severe toxicity observed compared to neutropenia and thrombocytopenia in both arms. Nadir was mostly noticed in the third week of the treatment that was closely monitored by repeating blood counts twice weekly. Patients with low counts were managed using antibiotics and antifungals. One patient in the three-weekly arm developed platelet counts below 35,000 and was then transfused with platelets. Grade III and IV toxicities were less in our study.

The upper gastrointestinal toxicity in the form of nausea and vomiting was statistically higher in the tri-weekly arm $(4.9 \%$ vs. $0.9 \%, \mathrm{p}=0.03)$ and was managed using 5-hydroxytryptamine3 blockers. In case of uncontrolled symptoms, levosulpiride (a class of benzamides) was added. Grades 1 and 2 lower gastrointestinal toxicity was significantly higher in the three-weekly arm $(42.7 \%$ vs. $27.5 \%$, $\mathrm{p}=0.04$ ) and was managed conservatively. Serum sodium and potassium levels were regularly monitored and corrected as and when required. The late toxicities of the lower gastrointestinal tract were also higher in the three-weekly arm with no statistical significance.

\section{Discussion}

The introduction of concurrent chemotherapy to radical radiotherapy in the treatment of carcinoma cervix has evolved over the last three decades. The National Cancer Institute, United States, in 1999 announced: "strong consideration should be given in incorporation of concurrent cisplatin-based chemotherapy with radiotherapy in women who require radiotherapy in the treatment of cervical cancer" based on five randomized clinical trials [5, 18-22]. In the meta-analysis from the MRC trials group, UK, cisplatin attempted alone or in combination (Hydroxyurea, 5Fu, Mitomycin C) improved the local control with acceptable toxicities. 
Table 1. Patient characteristics

\begin{tabular}{|c|c|c|c|c|}
\hline \multicolumn{3}{|l|}{ Characteristics } & Arm A (109) & Arm B (103) \\
\hline \multirow{2}{*}{ Age } & \multicolumn{2}{|c|}{ Median } & 45 & 45 \\
\hline & \multicolumn{2}{|c|}{ Range } & $23-67$ & $35-65$ \\
\hline \multirow{2}{*}{ FIGO stage } & \multicolumn{2}{|c|}{$\mathrm{IIIB}$} & 52 & 46 \\
\hline & \multicolumn{2}{|c|}{ IIB } & 57 & 57 \\
\hline \multirow{2}{*}{ Radiotherapy plan } & \multicolumn{2}{|c|}{ Two Fields } & 80 & 86 \\
\hline & \multicolumn{2}{|c|}{ Four Fields } & 29 & 17 \\
\hline \multirow{2}{*}{ Brachytherapy } & ICBT & LDR & 97 & 88 \\
\hline & ISBT & HDR & 12 & 15 \\
\hline OTT & \multicolumn{2}{|c|}{ Mean } & 50.02 & 50.07 \\
\hline \multirow{5}{*}{ Chemotherapy cycles } & \multicolumn{2}{|c|}{ One } & 3 & 22 \\
\hline & \multicolumn{2}{|c|}{ Two } & 7 & 81 \\
\hline & \multicolumn{2}{|c|}{ Three } & 27 & 0 \\
\hline & \multicolumn{2}{|c|}{ Four } & 46 & 0 \\
\hline & \multicolumn{2}{|c|}{ Five } & 26 & 0 \\
\hline Cumulative chemo dose [mg] & \multicolumn{2}{|c|}{ Mean } & $227.16( \pm 56.9)$ & $262.62( \pm 72.3)$ \\
\hline Point A dose & \multicolumn{2}{|c|}{ Mean } & $28.1( \pm 3.84)$ & $27.9( \pm 4.15)$ \\
\hline Point $A$ dose rate & \multicolumn{2}{|c|}{ Median } & 148.9 & 148.6 \\
\hline Bladder dose & \multicolumn{2}{|c|}{ Median } & 19.9 & 19.3 \\
\hline Bladder dose rate & \multicolumn{2}{|c|}{ Median } & 99.6 & 97.2 \\
\hline Rectal dose & \multicolumn{2}{|c|}{ Median } & 15.5 & 15.8 \\
\hline Rectal dose rate & \multicolumn{2}{|c|}{ Median } & 80.9 & 79.4 \\
\hline
\end{tabular}

FIGO — International Federation of Gynecology and Obstetrics; ICBT — intracavitary brachytherapy; ISBT — interstitial brachytherapy; LDR — low dose rate brachytherapy; HDR — high dose rate brachytherapy; OTT — overall treatment time

Table 2. Comparison of toxicities in weekly and three weekly arms

\begin{tabular}{|c|c|c|c|c|}
\hline Reactions & Grade & $\begin{array}{l}\text { Arm A } \\
N(\%)\end{array}$ & $\begin{array}{l}\text { Arm B } \\
N(\%)\end{array}$ & p-value \\
\hline \multirow{2}{*}{ Leucopenia } & Low & $57(52)$ & $63(61)$ & \multirow{2}{*}{0.574} \\
\hline & High & $5(4.5)$ & $8(7.7)$ & \\
\hline \multirow{2}{*}{ Neutropenia } & Low & $48(44)$ & 45 (43.7) & \multirow{2}{*}{0.804} \\
\hline & High & $5(4.6)$ & $3(2.9)$ & \\
\hline \multirow{2}{*}{ Thrombocytopenia } & Low & $18(16.5)$ & $18(17.5)$ & \multirow{2}{*}{0.8} \\
\hline & High & $1(0.9)$ & $2(1.9)$ & \\
\hline \multirow{2}{*}{ UGI } & Low & $45(41.2)$ & $54(52.4)$ & \multirow{2}{*}{0.03} \\
\hline & High & $1(0.9)$ & $5(4.9)$ & \\
\hline \multirow{2}{*}{ LGI } & Low & $30(27.5)$ & $44(42.7)$ & \multirow{2}{*}{0.047} \\
\hline & High & $1(0.9)$ & 0 & \\
\hline \multirow{2}{*}{ Cystitis } & Low & 0 & 0 & \multirow{2}{*}{0.234} \\
\hline & High & 0 & $2(1.9)$ & \\
\hline \multirow{2}{*}{ Proctitis } & Low & $8(7.3)$ & $10(9.7)$ & \multirow{2}{*}{0.634} \\
\hline & High & $4(3.7)$ & $2(1.9)$ & \\
\hline
\end{tabular}

UGI — upper gastrointestinal; LGI — lower gastrointestinal; Low — grade 1 and 2; High — grade 3 and 4; $\mathrm{N}$ - number of patients 
Runowicz et al. in 1989 evaluated the toxicity and potential synergism of concurrent chemoradiation with cisplatin, in which they used cisplatin $20 \mathrm{mg} / \mathrm{m}^{2}$ for 5 days, every 21 days, up to 2-5 cycles (concurrently and continued adjuvant cycles after teletherapy and brachytherapy) [23]. The median dose of cisplatin was $655 \mathrm{mg}$ with a median of four cycles of chemotherapy. Only $34 / 43$ patients were evaluable as four were discontinued owing to severe myelosuppression and three due to creatinine levels $>3 \mathrm{mg} \%$. Although the haematological toxicities, like leucopenia and neutropenia, observed in the study were severe, the treatment was well tolerated in the remaining patients by temporarily suspending therapy and supporting transfusions when necessary, the high incidence of the haematological reactions might be due to the difference in the median dose compared to our study.

The cytotoxic dose of $100 \mathrm{mg} / \mathrm{m}^{2}$ in the tri-weekly arm in our study was considered owing to the higher peak concentration of cisplatin possibly more critical in enhancing the efficacy of chemoradiation than weekly cisplatin. In addition, this dose schedule may be more effective in eliminating micro-metastasis resulting in decreased local failure, distant metastasis, and improving survival $[7,9]$.

Souhami et al. in 1993 used weekly cisplatin $30 \mathrm{mg} / \mathrm{m}^{2}$ alone along with radiotherapy in stage IIB-IVA carcinoma cervix in 50 patients, demonstrated that there was a high incidence of acute upper gastrointestinal toxicity of $50 \%$ and only $2 \%$ Grade III leucopoenia [24]. It was seen that there was a high incidence of late rectal reactions requiring intervention due to the high cumulative rectal dose. The difference in the dose of the weekly cisplatin $/ \mathrm{m}^{2}$ signifies the difference in the toxicities encountered by us.

The study reported by Ryu et al. in 2011 compared concurrent weekly and three-weekly regimens of cisplatin at $40 \mathrm{mg} / \mathrm{m}^{2}$ for six cycles and 75 $\mathrm{mg} / \mathrm{m}^{2}$ for three cycles, respectively ${ }^{7}$. The cisplatin regimen used in their study was almost similar, except that the three-weekly doses were $100 \mathrm{mg} / \mathrm{m}^{2}$ in our study. The analysis revealed a significant difference in neutropenia in both arms with high incidence in the weekly arm $(\mathrm{p}=0.03)$; however, no significant difference in other toxicities, like gastrointestinal, renal, and neurosensory toxicities
( $p>0.05)$, which projected the conclusion that the three-weekly arm had better tolerance in terms of toxicity and 5-year overall survival than the weekly $\mathrm{arm}$. There was no statistical difference in treatment completion rates of $86.3 \%$ and $92.5 \%$ in weekly and tri-weekly arms, respectively. On the contrary, our study showed significantly higher upper and lower gastro-intestinal toxicity and statistically insignificant haematological toxicity.

A meta-analysis by Yan $\mathrm{Hu}$ et al. published in 2012 evaluated the results of seven randomized studies conducted between 1995-2011 and observed lower leucopenia and thrombocytopenia in weekly than tri-weekly cisplatin which was similar to the findings of our study [25]. In their study, they did not find statistically significant differences in neuropathy and GI toxicity. Moreover, patients' preference to receive chemotherapy at 3 weeks intervals was flexible and convenient. Although weekly cisplatin shows lower risk haematologic toxicity with concurrent chemoradiation, there was no difference in PFS and OS between the two groups. Owing to increased toxicity associated with tri-weekly concurrent chemoradiation, weekly cisplatin chemotherapy was considered a more useful concurrent adjuvant chemoradiation regimen after radical surgery, recommending further clinical trials to confirm.

A meta-analysis of eight studies (six randomized and two retrospective) comparing tri-weekly vs. weekly cisplatin-based chemoradiation published between 1990 to Dec 2017 by Zu et al., found that tri-weekly CDDP concurrent with radiation showed better compliance [26]. No significant difference was observed between the two arms to acute adverse effects. Weekly cisplatin regimen was associated with a lower risk of leucopenia (odds ratio, 0.30 ; $95 \%$ confidence interval: $0.10-0.92$; $\mathrm{p}=0.03)$ similar to our study. In this meta-analysis, single-agent tri-weekly CDDP $\left(20 \mathrm{mg} / \mathrm{m}^{2}\right.$ for 5 days or $75 \mathrm{mg} / \mathrm{m}^{2}$ ) was associated with better compliance compared to weekly cisplatin $\left(40 \mathrm{mg} / \mathrm{m}^{2}\right)$ with concurrent radiation in patients with locally advanced cervical cancer. The incidence of haematological toxicity was higher in the tri-weekly cisplatin arm, which is similar to the findings of a previous meta-analysis and our study also showed identical results. Finally, the authors recommend a tri-weekly over weekly cisplatin regimen for concurrent chemoradiation arm in patients with locally 
advanced cervical cancer given the better response of the disease.

In our study, all patients completed planned dose of radiation (teletherapy and brachytherapy), whereas $66.07 \%$ in weekly and $78.64 \%$ in tri-weekly patients received planned dose of chemotherapy. This was owing to acute toxicities, namely leucopenia $\left(0.7-3.8 \times 10^{9} / \mathrm{L}\right)$, neutropenia $\left(0.5-1.5 \times 10^{9} / \mathrm{L}\right)$, and thrombocytopenia $\left(33-98 \times 10^{9} / \mathrm{L}\right)$. The comparison of these toxicities in both arms was not statistically significant. As we aimed to assess the tolerance to the treatment, none of the patients received any granulocyte colony-stimulating factors for improving blood counts. The majority of the patients recovered the counts within 2-3 weeks.

The acute upper gastro-intestinal toxicity grade I and II observed in our study was $52.4 \%$ in the tri-weekly arm and $41.2 \%$ in weekly, which was statistically significant $(\mathrm{p}=0.03)$, and acute lower gastro-intestinal grade I \& II reactions were $42.7 \%$ in the tri-weekly arm versus $27.5 \%$ in the weekly arm just reaching statistical significance $(\mathrm{p}=0.047)$. Although late rectal reactions (proctitis) were higher in the tri-weekly arm, they did not reach statistical significance. All the toxicities were managed conservatively.

\section{Conclusion}

Our study showed that toxicities and compliance in both arms were comparable. Tri-weekly cisplatin-based concurrent chemoradiation was associated with statistically non-significant haematological toxicity; however, significant acute upper and lower gastrointestinal toxicity, which was manageable conservatively. To conclude, in high-volume centres like ours, the use of tri-weekly cisplatin is feasible with good compliance and acceptable toxicities. However, further randomized controlled trials with large numbers are required to recommend the results.

\section{Acknowledgements}

We acknowledge all our team members who are involved in patient care and management of the recruited patients. We also thank Dr. Jagadeesan. $\mathrm{P}$ for his contribution to statistical analysis. We also thank Dr. Manjula. M.V for being helpful for the compilation of the data.

\section{Conflict of interest}

None declared.

\section{Funding}

None declared.

\section{References}

1. Bray F, Ferlay J, Soerjomataram I, et al. Global cancer statistics 2018: GLOBOCAN estimates of incidence and mortality worldwide for 36 cancers in 185 countries. CA Cancer J Clin. 2018; 68(6): 394-424, doi: 10.3322/ caac.21492, indexed in Pubmed: 30207593.

2. Aruna Rastogi; National Health Portal India. www.nhp.gov. in/disease/reproductive-system/female-gynaecologicaldiseases-/cervical-cancer (Aug 2016).

3. Chemoradiotherapy for Cervical Cancer Meta-Analysis Collaboration. Reducing uncertainties about the effects of chemoradiotherapy for cervical cancer: a systematic review and meta-analysis of individual patient data from 18 randomized trials. J Clin Oncol. 2008; 26(35): 5802-5812, doi: 10.1200/JCO.2008.16.4368, indexed in Pubmed: 19001332.

4. Tzioras S, Pavlidis N, Paraskevaidis E, et al. Effects of different chemotherapy regimens on survival for advanced cervical cancer: systematic review and meta-analysis. Cancer Treat Rev. 2007; 33(1): 24-38, doi: 10.1016/j. ctrv.2006.09.007, indexed in Pubmed: 17112673.Keys HM, Bundy BN, Stehman FB, et al. Cisplatin, radiation, and adjuvant hysterectomy compared with radiation and adjuvant hysterectomy for bulky stage IB cervical carcinoma. N Engl J Med. 1999; 340(15): 1154-1161, doi: 10.1056/NEJM199904153401503, indexed in Pubmed: 10202166.

5. Gaffney DK, Erickson-Wittmann BA, Jhingran A, et al. ACR Appropriateness Criteria ${ }^{\circledR}$ on Advanced Cervical Cancer Expert Panel on Radiation Oncology-Gynecology. Int J Radiat Oncol Biol Phys. 2011; 81(3): 609-614, doi: 10.1016/j. ijrobp.2010.11.005, indexed in Pubmed: 21215531.

6. Ryu SY, Lee WM, Kim K, et al. Randomized clinical trial of weekly vs. triweekly cisplatin-based chemotherapy concurrent with radiotherapy in the treatment of locally advanced cervical cancer. Int J Radiat Oncol Biol Phys. 2011;81(4): e577-e581, doi: 10.1016/j.jirobp.2011.05.002, indexed in Pubmed: 21840137.

7. Lee HN, Lee KHo, Lee DW, et al. Weekly cisplatin therapy compared with triweekly combination chemotherapy as concurrent adjuvant chemoradiation therapy after radical hysterectomy for cervical cancer. Int J Gynecol Cancer. 2011; 21(1): 128-136, doi: 10.1097/IGC.0b013e318200f7c5, indexed in Pubmed: 21330837.

8. Bonomi P, Blessing JA, Stehman FB, et al. Randomized trial of three cisplatin dose schedules in squamous-cell carcinoma of the cervix: a Gynecologic Oncology Group study. J Clin Oncol. 1985; 3(8): 1079-1085, doi: 10.1200/ JCO.1985.3.8.1079, indexed in Pubmed: 3894589.

9. Serkies K, Jassem J. Concurrent weekly cisplatin and radiotherapy in routine management of cervical cancer: a report on patient compliance and acute toxicity. Int J Ra- 
diat Oncol Biol Phys. 2004; 60(3): 814-821, doi: 10.1016/j. ijrobp.2004.04.042, indexed in Pubmed: 15465198.

10. Adelstein DJ, Li Yi, Adams GL, et al. An intergroup phase III comparison of standard radiation therapy and two schedules of concurrent chemoradiotherapy in patients with unresectable squamous cell head and neck cancer. J Clin Oncol. 2003; 21(1): 92-98, doi: 10.1200/JCO.2003.01.008, indexed in Pubmed: 12506176.

11. Bernier J, Domenge C, Ozsahin M, et al. European Organization for Research and Treatment of Cancer Trial 22931. Postoperative irradiation with or without concomitant chemotherapy for locally advanced head and neck cancer. N Engl J Med. 2004; 350(19): 1945-1952, doi: 10.1056/ NEJMoa032641, indexed in Pubmed: 15128894.

12. Cooper JS, Pajak TF, Forastiere AA, et al. Radiation Therapy Oncology Group 9501/Intergroup. Postoperative concurrent radiotherapy and chemotherapy for high-risk squamous-cell carcinoma of the head and neck. N Engl J Med. 2004; 350(19): 1937-1944, doi: 10.1056/NEJMoa032646, indexed in Pubmed: 15128893.

13. Fountzilas G, Skarlos D, Kosmidis $P$, et al. Radiation therapy and concurrent cisplatin administration in locally advanced head and neck cancer. A Hellenic Cooperative Oncology Group study. Acta Oncol. 1994; 33(7): 825-830, doi: 10.3109/02841869409083955, indexed in Pubmed: 7993653.

14. Nagy VM, Ordeanu C, Coza O, et al. Randomized phase 3 trial comparing 2 cisplatin dose schedules in 326 patients with locally advanced squamous cell cervical carcinoma: long-term follow-up. Int J Gynecol Cancer. 2012; 22(9): 1538-1544, doi: 10.1097/IGC.0b013e318270590a, indexed in Pubmed: 23070071.

15. Kim YS, Shin SS, Nam JH, et al. Prospective randomized comparison of monthly fluorouracil and cisplatin versus weekly cisplatin concurrent with pelvic radiotherapy and high-dose rate brachytherapy for locally advanced cervical cancer. Gynecol Oncol. 2008; 108(1): 195-200, doi: 10.1016/j.ygyno.2007.09.022, indexed in Pubmed: 17963825.

16. Rose PG, Ali S, Watkins E, et al. Gynecologic Oncology Group. Long-term follow-up of a randomized trial comparing concurrent single agent cisplatin, cisplatin-based combination chemotherapy, or hydroxyurea during pelvic irradiation for locally advanced cervical cancer: a Gynecologic Oncology Group Study. J Clin Oncol. 2007; 25(19): 2804-2810, doi: 10.1200/JCO.2006.09.4532, indexed in Pubmed: 17502627.

17. Whitney CW, Sause W, Bundy BN, et al. Randomized comparison of fluorouracil plus cisplatin versus hy- droxyurea as an adjunct to radiation therapy in stage IIB-IVA carcinoma of the cervix with negative para-aortic lymph nodes: a Gynecologic Oncology Group and Southwest Oncology Group study. J Clin Oncol. 1999; 17(5): 1339-1348, doi: 10.1200/JCO.1999.17.5.1339, indexed in Pubmed: 10334517.

18. Morris M, Eifel PJ, Lu J, et al. Pelvic radiation with concurrent chemotherapy compared with pelvic and para-aortic radiation for high-risk cervical cancer. $\mathrm{N}$ Engl J Med. 1999; 340(15): 1137-1143, doi: 10.1056/ NEJM199904153401501, indexed in Pubmed: 10202164.

19. Rose PG, Bundy BN, Watkins EB, et al. Concurrent cisplatin-based radiotherapy and chemotherapy for locally advanced cervical cancer. N Engl J Med. 1999; 340(15): 1144-1153, doi: 10.1056/NEJM199904153401502, indexed in Pubmed: 10202165.

20. Peters WA, Liu PY, Barrett RJ, et al. Concurrent chemotherapy and pelvic radiation therapy compared with pelvic radiation therapy alone as adjuvant therapy after radical surgery in high-risk early-stage cancer of the cervix. J Clin Oncol. 2000; 18(8): 1606-1613, doi: 10.1200/ JCO.2000.18.8.1606, indexed in Pubmed: 10764420.

21. Pearcey $R$, Brundage $M$, Drouin $P$, et al. Phase III trial comparing radical radiotherapy with and without cisplatin chemotherapy in patients with advanced squamous cell cancer of the cervix. J Clin Oncol. 2002; 20(4): 966-972, doi: 10.1200/JCO.2002.20.4.966, indexed in Pubmed: 11844818.

22. Runowicz CD, Wadler $S$, Rodriguez-Rodriguez $L$, et al. Concomitant cisplatin and radiotherapy in locally advanced cervical carcinoma. Gynecol Oncol. 1989; 34(3): 395-401, doi: 10.1016/0090-8258(89)90180-7, indexed in Pubmed: 2767531.

23. Clark BG, Souhami L, Roman TN, et al. Weekly cisplatin plus external beam radiotherapy and high dose rate brachytherapy in patients with locally advanced carcinoma of the cervix. Int J Radiat Oncol Biol Phys. 1993; 27(4): 871-878, doi: 10.1016/0360-3016(93)90462-5, indexed in Pubmed: 8244817.

24. Hu Y, Cai ZQ, Su XY. Concurrent weekly cisplatin versus triweekly cisplatin with radiotherapy in the treatment of cervical cancer: a meta-analysis result. Asian Pac J Cancer Prev. 2012; 13(9): 4301-4304, doi: 10.7314/apjcp.2012.13.9.4301, indexed in Pubmed: 23167332.

25. Zhu J, Ji S, Hu Q, et al. Concurrent weekly single cisplatin vs triweekly cisplatin alone with radiotherapy for treatment of locally advanced cervical cancer: a meta-analysis. Cancer Manag Res. 2018; 10: 1975-1985, doi: 10.2147/CMAR. S167938, indexed in Pubmed: 30034252. 